\title{
A CMOS-compatible morphotropic phase boundary
}

\author{
Alireza Kashir*, Hyunsang Hwang* \\ Center for Single Atom-based Semiconductor Device and Department of Materials Science and \\ Engineering, Pohang University of Science and Technology (POSTECH), Pohang, Republic of Korea \\ *Email: kashir@postech.ac.kr, hwanghs@postech.ac.kr
}

\begin{abstract}
Morphotropic phase boundaries (MPBs) show substantial piezoelectric and dielectric responses, which have practical applications. The predicted existence of $\mathrm{MPB}$ in $\mathrm{HfO}_{2}-\mathrm{ZrO}_{2}$ solid solution thin film has provided a new way to increase the dielectric properties of a silicon-compatible device. Here, we present a new fabrication design by which the density of MPB $\rho_{M P B}$ and consequently the dielectric constant $\epsilon_{\mathrm{r}}$ of $\mathrm{HfO}_{2}-\mathrm{ZrO}_{2}$ thin film was considerably increased. The $\rho_{M P B}$ was controlled by fabrication of a $10-\mathrm{nm}\left[1 \mathrm{~nm}-\mathrm{Hf}_{0.5} \mathrm{Zr}_{0.5} \mathrm{O}_{2}\right.$ (Ferroelectric)/ $1 \mathrm{~nm}-\mathrm{ZrO}_{2}$ (Antiferroelectric)] nanolaminate followed by an appropriate annealing process. The coexistence of orthorhombic and tetragonal structures, which are the origins of ferroelectric (FE) and antiferroelectric (AFE) behaviors, respectively, was structurally confirmed, and a double hysteresis loop that originates from AFE ordering, with some remnant polarization that originates from FE ordering, was observed in P-E curve. A remarkable increase in $\epsilon_{\mathrm{r}}$ compared to the conventional $\mathrm{HfO}_{2}-\mathrm{ZrO}_{2}$ thin film was achieved by controlling the FE-AFE ratio. The fabrication process was performed at low temperature $\left(250{ }^{\circ} \mathrm{C}\right)$ and the device is compatible with silicon technology, so the new design yields a device that has possible applications in near-future electronics.
\end{abstract}

Keywords: High- $\kappa$ dielectrics; $\mathrm{HfO}_{2} ; \mathrm{ZrO}_{2} ; \mathrm{CMOS}$; Equivalent oxide thickness; 


\section{Introduction}

The downscaling of Complementary Metal-Oxide-Semiconductor (CMOS) technology is approaching the limit to reduction in the physical thickness of gate dielectric, without incurring excessive leakage current. However, high- $\kappa$ metal gate transistors require the reduction in equivalent oxide thickness (EOT) of the gate dielectric. Materials with high dielectric permittivity $\epsilon_{r}$ may enable achievement of this goal. EOT as small as $3.5-4 \AA$ has been achieved by using new high $\epsilon_{r}$ materials [1-4], but they usually have a low band gap $E_{\mathrm{g}}$, and their dielectric properties decrease significantly in thin films, so the task of obtaining satisfactory $\epsilon_{r}$ in the acceptable thickness range is difficult. Moreover, these materials are mainly incompatible with silicon electronics, or contain toxic elements, and are therefore not appropriate for the current electronics industry.

Many research groups have attempted to reach the ideal region in the graph of $E_{\mathrm{g}}$ versus $\epsilon_{\mathrm{r}}$ [5] by increasing of the dielectric properties of silicon-compatible materials. Methods tried include adding dopants [6-8], stabilizing the metastable high $\epsilon_{r}$ phase [9], designing nanolaminates [1011], and preventing formation of an interfacial region [12-13]. Despite the considerable improvements, these attempts seem to have reached their limits, so new approaches must be developed.

Theoretical prediction that morphotropic phase boundaries (MPBs) can form in $\mathrm{HfO}_{2}-\mathrm{ZrO}_{2}$ solid solution [14] raises the possibility that ceramic engineering may enable development of lead-free and silicon-compatible high- $\kappa$ dielectric without any degradation in $E_{\mathrm{g}}$, because the chemistries of hafnium and zirconium are more nearly identical than are those of any other two transition metals. Moreover, they have wide band gap $(\sim 5.5 \mathrm{eV})$, extreme thinness, good reliability, high binding energy between the oxygen and transition metal ions, and are compatible with CMOS processes. Furthermore, use of $\mathrm{ZrO}_{2}$ and $\mathrm{HfO}_{2}$ in the semiconductor fabrication process is in the mature stage. An MPB is a region of coexisting phases in the phase diagram of ferroelectrics [15-16]. The MPB separates two competing phases that have distinct symmetries. Realizing MPB usually requires a flat energy surface between different symmetries; this surface facilitates polarization rotation that arises from the coupling between two equivalent energy states, and thus induces large dielectric and piezoelectric responses as a result of enhanced polarizability [17-20]. The MPB has potential 
applications because the variable that drives the transition (i.e., compositions) is inherent, so the engineering of a ferroelectric (FE) system near MPB may further boost the $\epsilon_{r}$ without degrading the band-gap.

The existence of MPB has been predicted in $\mathrm{Hf}_{1-x} \mathrm{Zr}_{x} \mathrm{O}_{2}(\mathrm{HZO})$ system [14], but the coexistence of tetragonal (t) and orthorhombic (o) phases is substantially affected by fabrication conditions [2125] (e.g., film thickness, electrode materials, grain size, point defects, annealing conditions) and the energy landscape cannot be simply predicted or controlled. Even though the existence of MPB was predicted at $x=0.7$ ( $\mathrm{Ni}$ et al. [14]), the increase in $\epsilon_{\mathrm{r}}$ was observed experimentally at $x \approx 0.5$ (Park et al. [26]).

Here, we introduce a new design of $\mathrm{HfO}_{2}-\mathrm{ZrO}_{2}$ thin film to control and maximize the formation of MPB regions. The $\mathrm{ZrO}_{2}$ thin film shows antiferroelectric (AFE) behavior that originates from a t(nonpolar) to o- (polar) structural transition induced by the electric field [27]. In contrast, HZO ( $x$ $=0.5)$ thin film can exhibit FE properties by stabilization of the metastable non-centrosymmetric o-phase during the thin-film fabrication process [28]. The occurrence of these traits suggests that a nanolaminate of $\mathrm{HZO}(x=0.5)(\mathrm{FE})$ and $\mathrm{ZrO}_{2}(\mathrm{AFE})$ followed by an optimum annealing process may lead to formation of MPB at interfacial regions, and yield in a strong dielectric response.

\section{Experiments}

Three different types of metal-insulator-metal (MIM) capacitors were fabricated on $\mathrm{SiO}_{2} / \mathrm{Si}$ substrate. They were $\mathrm{W} / \mathrm{ZrO}_{2} / \mathrm{W}, \mathrm{W} / \mathrm{HZO} / \mathrm{W}$ and $\mathrm{W} /\left[\mathrm{HZO}(1 \mathrm{~nm}) / \mathrm{ZrO}_{2}(1 \mathrm{~nm})\right] \times 5 / \mathrm{W}$ $(\mathrm{W} / \mathrm{HZZ} / \mathrm{W})$. In all three devices the insulator layer was $~ 10 \mathrm{~nm}$ thick. The $\mathrm{W}$ bottom and top electrodes were deposited using rf-sputtering at room temperature (RT). The $\mathrm{HZO}$ and $\mathrm{ZrO}_{2}$ thin films were deposited using atomic layer deposition (ALD). The Hf precursor was tetrakis (ethylmethylamido) hafnium (IV) ( $\left.\mathrm{Hf}\left[\mathrm{N}-\left(\mathrm{C}_{2} \mathrm{H}_{5}\right) \mathrm{CH}_{3}\right]_{4}\right)$; the $\mathrm{Zr}$ precursor was Tetrakis (ethylmethylamido) zirconium (IV) $\left(\mathrm{Zr}\left[\mathrm{N}-\left(\mathrm{C}_{2} \mathrm{H}_{5}\right) \mathrm{CH}_{3}\right]_{4}\right)$. The oxidant was $\mathrm{O}_{3}\left(276 \mathrm{~g} / \mathrm{Nm}^{3}\right)$. During the growth of $\mathrm{HZO}$, the $\mathrm{HfO}_{2}$ and $\mathrm{ZrO}_{2}$ layers were deposited at a $\sim 1: 1$ cycle ratio to form $\mathrm{Hf}_{0.5} \mathrm{Zr}_{0.5} \mathrm{O}_{2}$ layers. An ozone pulse duration of $15 \mathrm{~s}$ was applied for each deposition; this duration appeared to be an optimum dosage to remove carbon contaminants from the deposited materials at a given condition [29]. The substrate temperature was maintained constant at $250{ }^{\circ} \mathrm{C}$ during deposition of all insulating films presented in this study, and the precursors' temperature was kept 
at $90{ }^{\circ} \mathrm{C}$. The growth rates of $\mathrm{HfO}_{2}$ and $\mathrm{ZrO}_{2}$ were almost identical at $\sim 1 \AA$ /cycle. The number of $\mathrm{HfO}_{2}$ and $\mathrm{ZrO}_{2}$ cycles required to deposit $\left(1 \mathrm{~nm} \mathrm{HZO} / 1 \mathrm{~nm} \mathrm{ZrO}_{2}\right) \times 5$ nanolaminates was determined by considering these growth rates. Top electrodes with sizes from $30 \times 30 \mu \mathrm{m}^{2}$ to 100 $\times 100 \mu \mathrm{m}^{2}$ were patterned using a lift-off process by a photolithography method. Finally, the capacitors with different electrode areas were annealed under $1000 \mathrm{sccm}$ high-purity $\mathrm{N}_{2}$ flow at $500{ }^{\circ} \mathrm{C}$ for $30 \mathrm{sec}$. Moreover, the $\mathrm{W} / \mathrm{HZZ} / \mathrm{W}$ devices have gone through variety of annealing conditions from $300{ }^{\circ} \mathrm{C}$ to $700{ }^{\circ} \mathrm{C}$ to achieve the optimum dielectric performance.

The crystal structures of the films were investigated using an X-ray diffractometer in grazing incidence geometry (GIXRD) and high-resolution and scanning transmission electron microscopy (HRTEM and STEM). To determine the elemental composition of the films, X-ray photoelectron spectroscopy (XPS) was performed using the depth-profiling mode by $\mathrm{Ar}^{+}$ion sputtering on asgrown uncapped samples (Figure S1). The ferroelectric properties of the devices were measured using an LCII ferroelectric precision tester (Radiant Technologies), and the capacitance vs electric field $(C-E)$ were evaluated using a Keysight B1500A semiconductor device parameter analyzer. The $\mathrm{C}-\mathrm{E}$ measurement was executed with an amplitude of $50 \mathrm{mV}$ at a frequency of $10 \mathrm{kHz}$. Rayleigh dielectric measurements were carried out at a frequency of $10 \mathrm{kHz}$, wherein the ac-field strength was ramped from $1 \mathrm{mV}$ to $2 \mathrm{~V}$. An Agilent E4980A Precision LCR Meter was used and the electric field was increased incrementally by $+5 \mathrm{mV}$ steps. The dielectric constants were extracted from capacitance values obtained from Rayleigh measurements. The measurements were conducted at RT.

\section{Results and discussion}

A schematic of the $\mathrm{W} / \mathrm{HZZ} / \mathrm{W}$ device and an atomic model are presented in figure $1 \mathrm{a}$ and $\mathrm{b}$, respectively. Recent phase-field modeling (Ni et al. [14]) studied the effect of $\mathrm{Zr}$ concentration $(x)$ on the total energy of HZO system. This modeling revealed the total energy of HZO system versus the sub-lattice polarization $\left(P_{1}, P_{2}\right)$. The orthorhombic FE phase is energetically favored if the energy minimum lies on the anti-diagonal line, where the sub-lattice polarizations equal each other $\left(P_{1}=P_{2}\right)$. In contrast, the tetragonal AFE phase is energetically favored if the energy minimum lies on the diagonal line, where the sub-lattice polarizations are opposite to each other $\left(P_{1}=-P_{2}\right)$. The simulations predicted that a composition between $\mathrm{Hf}_{0.5} \mathrm{Zr}_{0.5} \mathrm{O}_{2}$ and $\mathrm{ZrO}_{2}(0.5<x<1)$ would 
have a flat energy surface between different symmetries $\left(P_{1}=P_{2}\right.$ and $\left.P_{1}=-P_{2}\right)$ (as it is illustrated in the figure 1c). This flat energy surface facilitates the polarization rotation between $\mathrm{FE}$ and $\mathrm{AFE}$ orderings stimulated by small external electrical or mechanical stimuli and results in a substantial increase in dielectric response [14].

(a)

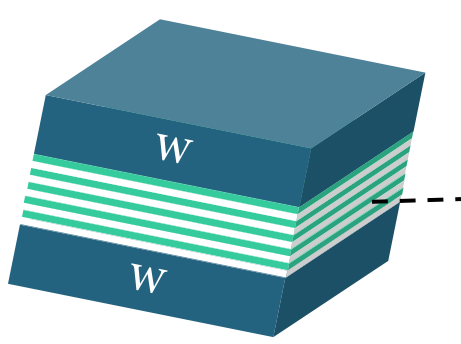

(d)

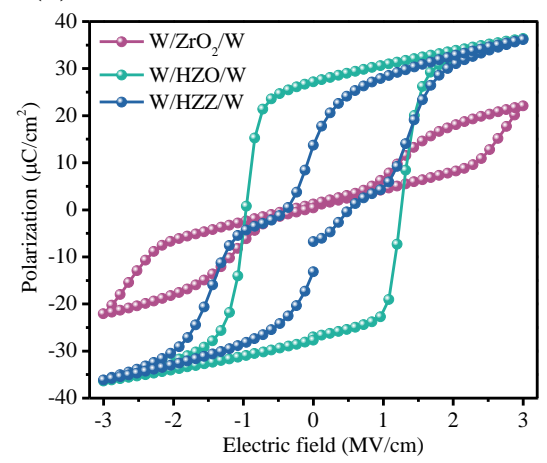

(b)

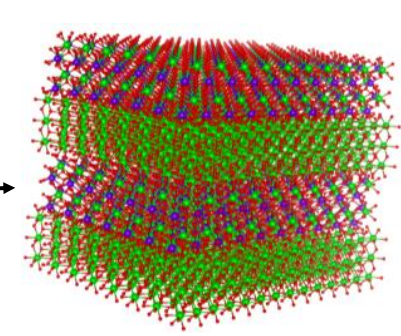

(e)

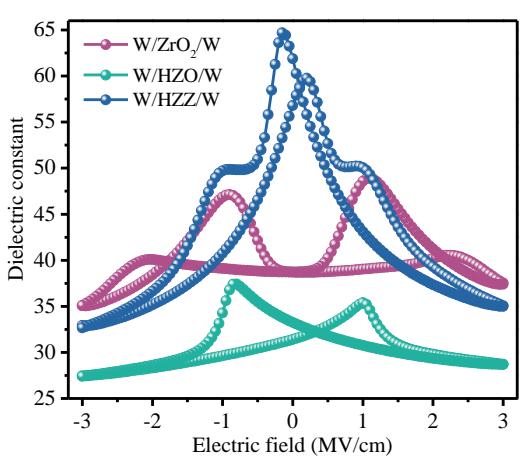

(c)
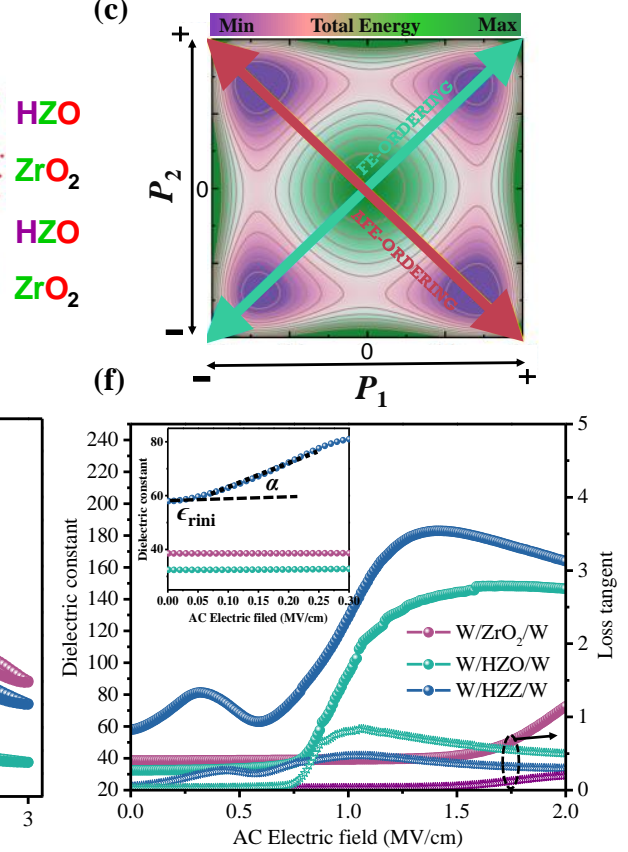

Figure 1. (a) Schematic of W/HZZ/W nanolaminate capacitor, (b) an atomic view of $\mathrm{HZO} / \mathrm{ZrO}_{2}$ supercell. Purple spheres: Hf, green: Zr, red: O. (c) Total energy contour on the sub-lattice polarization $\left(P_{1}, P_{2}\right)$ plane of $\mathrm{Zr}$-doped $\mathrm{HfO}_{2}$ system in a composition between $0.5<x<1$ [14]. (d) Polarization vs. electric field $E$, (e) dielectric constants vs. applied biased $E$ and (f) Rayleigh dielectric measurements for $\mathrm{W} / \mathrm{ZrO}_{2} / \mathrm{W}$, $\mathrm{W} / \mathrm{HZO} / \mathrm{W}$ and $\mathrm{W} / \mathrm{HZZ} / \mathrm{W}$ devices fabricated on $\mathrm{SiO}_{2} /$ Silicon substrates and annealed at $500{ }^{\circ} \mathrm{C}$ for $30 \mathrm{~s}$.

The curves of polarization versus electric field $(P-E)$ of $\mathrm{W} / \mathrm{ZrO}_{2} / \mathrm{W}, \mathrm{W} / \mathrm{HZO} / \mathrm{W}$ and $\mathrm{W} / \mathrm{HZZ} / \mathrm{W}$ devices annealed at $500{ }^{\circ} \mathrm{C}$ for $30 \mathrm{~s}$ (figure $1 \mathrm{~d}$ ) show that the $\mathrm{W} / \mathrm{ZrO}_{2} / \mathrm{W}$ device has $\mathrm{AFE}$ behavior with $2 P_{\mathrm{r}}=0$ and a double-hysteresis loop. The density functional theory calculations predicted a small energy difference of $\sim 1 \mathrm{meV} / \mathrm{f}$. $\mathrm{u}$. between the nonpolar $\mathrm{t}$ - and polar o-structure in $\mathrm{ZrO}_{2}$ [27]; this difference is characteristic of AFE. In contrast, the HZO sample has FE properties with $2 P_{\mathrm{r}}=54 \mu \mathrm{C} / \mathrm{cm}^{2}$ which is believed to originate from the metastable o-phase [28]. The W/HZZ/W device shows a combination of $\mathrm{FE}$ and AFE features with $2 P_{\mathrm{r}}=13 \mu \mathrm{C} / \mathrm{cm}^{2}$. The maximum polarization $P_{\max }$ of $\mathrm{HZZ}$ nanolaminate is almost the same as in $\mathrm{HZO}$, and is attributed to the 
electric field-induced reversible structural change from t- to o-phase. As the electric field was removed, the polarization dropped. A remnant polarization $P_{\mathrm{r}} \sim 6.5 \mu \mathrm{C} / \mathrm{cm}^{2}$ in $\mathrm{HZZ}$ film originated from the o-phase, which is non-centrosymmetric and thus ferroelectric. Ultrathin $\mathrm{Zr}$-doped $\mathrm{HfO}_{2}$ film with 1-nm thickness maintains its FE properties [30]. Thus, a combination of FE (mainly from $\mathrm{HZO}$ layers) and AFE (mainly from $\mathrm{ZrO}_{2}$ layers) emerged in $\mathrm{HZZ}$ nanolaminates. This behavior may introduce MPB regions at the FE/AFE interfacial areas. This fact was revealed by the capacitance - electrical field $(C-E)$ measurement, which shows a considerable increase in $\epsilon_{\mathrm{r}}$ of $\mathrm{HZZ}$ nanolaminate at bias electric field $E=0 \mathrm{MV} / \mathrm{cm}$ (figure 1e). The $\mathrm{HZO}$ and $\mathrm{ZrO}_{2}$ devices showed $\epsilon_{\mathrm{r}}-E$ behavior that agreed well with $\mathrm{FE}$ and AFE properties, respectively. At $E=0 \mathrm{MV} / \mathrm{cm}$, $\mathrm{HZZ}$ had $\epsilon_{\mathrm{r}}=60$ whereas $\mathrm{ZrO}_{2}$ thin film had $\epsilon_{\mathrm{r}}=39$, and $\mathrm{HZO}$ thin films had $\epsilon_{\mathrm{r}}=32$. Therefore, the nanolaminate design increased the $\epsilon_{\mathrm{r}}$ of $\mathrm{ZrO}_{2}-\mathrm{HfO}_{2}$ thin film by $54 \%$, and achieved the highest $\epsilon_{\mathrm{r}}$ yet reported for an $\mathrm{HfO}_{2}-\mathrm{ZrO}_{2}$ device. In $\mathrm{ZrO}_{2}$ devices the maximum $\epsilon_{\mathrm{r}}$ occurred before 0 $\mathrm{MV} / \mathrm{cm}$, as is characteristic of AFE behaviors, whereas in $\mathrm{HZO}$ devices the maximum $\epsilon_{\mathrm{r}}$ occurred after $0 \mathrm{MV} / \mathrm{cm}$, as is characteristic of FE behaviors. $\mathrm{HZZ}$ nanolaminate had maximum $\epsilon_{\mathrm{r}}$ near $E=$ $0 \mathrm{MV} / \mathrm{cm}$.

Rayleigh dielectric measurement of HZZ nanolaminate was performed to further investigate its dielectric behavior. The $\mathrm{HZO}$ and $\mathrm{ZrO}_{2}$ behaviors were presented as a reference (figure 1f). The Rayleigh measurement studies the behavior of FE materials in three different regimes: low, high, and switching fields [31-33]. In the low-field regime, an enhanced field-independent constant permittivity represented by the reversible Rayleigh parameter $\epsilon_{r_{i n i}}$ indicates large intrinsic lattice and reversible motion of the interface around its equilibrium position along the walls of the potential energy well. In the high field (Rayleigh regime), a steep linear slope indicated by the Rayleigh coefficient $\alpha$ is typical of increased irreversible displacement of domain walls or phase boundaries from one potential energy well to another, and acquiring a new position. Thus, the overall dielectric response in the Rayleigh region is represented by [33]

$$
\epsilon_{r}=\epsilon_{r_{i n i}}+\alpha E_{0}
$$

where $E_{0}$ is the $\mathrm{AC}$ electric field amplitude. The Rayleigh parameters $\left(\epsilon_{r_{\text {ini }}}\right.$ and $\alpha$ ) for nanolaminate device are presented in the inset of figure $1 \mathrm{f}$. 
The $\mathrm{ZrO}_{2}$ had a constant $\epsilon_{\mathrm{r}}$ at $E<1.5 \mathrm{MV} / \mathrm{cm}$, as expected from the tetragonal non-polar structure (Figure 1f). $\epsilon_{\mathrm{r}}$ increased sharply at $E>1.5 \mathrm{MV} / \mathrm{cm}$; this behavior can be attributed to formation of o-phase under a strong electric field. Therefore, a feature similar to the FE system (i.e., HZO) can be observed in $\mathrm{ZrO}_{2}$ film at relatively high $E$. $\mathrm{HZO}$ film had $\epsilon_{r_{\text {ini }}} \sim 33$, which is smaller than that of $\mathrm{ZrO}_{2}\left(\epsilon_{r_{\text {ini }}}=39\right.$, (Table 1)). The difference can be understood considering the formation of ophase in $\mathrm{Zr}$-doped $\mathrm{HfO}_{2} . \epsilon_{\mathrm{r}}$ is higher in the t-phase than in the o-phase [34].

The HZO film had a small $\alpha=118 \mu \mathrm{m} / \mathrm{kV}$, because the film has a low domain-wall mobility [31]. At $E>750 \mathrm{kV} / \mathrm{cm}$, a sharp increase of $\epsilon_{\mathrm{r}}$ can be observed which corresponds to the onset of switching.

Table 1. The values of $\epsilon_{r_{i n i}}$ (dielectric constant at low-field regime) and $\alpha[\mu \mathrm{m} / \mathrm{kV}]$ for different devices extracted from the Rayleigh dielectric measurements (Figure 1f).

\begin{tabular}{|c|c|c|c|}
\hline & \multicolumn{3}{|c|}{ Sample } \\
\hline Parameter & $\mathrm{ZrO}_{2}$ & $\mathrm{HZO}$ & $\mathrm{HZZ}$ \\
\hline $\boldsymbol{\epsilon}_{\boldsymbol{r i n i}_{i n}}$ & 39 & 33 & 60 \\
\hline $\boldsymbol{\alpha}$ & 0 & 118 & 847 \\
\hline
\end{tabular}

The HZZ nanolaminate had $\epsilon_{r_{i n i}} \sim 60$, which is considerably higher than that of $\mathrm{HZO}$ and $\mathrm{ZrO}_{2}$. Moreover, in the initial step of the Rayleigh dielectric measurement of HZZ nanolaminate, $\epsilon_{\mathrm{r}}$ increased steeply from 60 to 80 at $E=300 \mathrm{kV} / \mathrm{cm}$; this is twice the $\epsilon_{\mathrm{r}}$ of $\mathrm{t}-\mathrm{ZrO}_{2}$ thin film.

The HZZ nanolaminate shows two peaks in its dielectric response, whereas $\mathrm{HZO}$ and $\mathrm{ZrO}_{2}$ show only one. A non-uniform distribution of the energy barriers causes a smooth blending between the three regions; this observation suggests that reducing the extent of the Rayleigh region, the increase of $\epsilon_{\mathrm{r}}$ at the onset of increased amplitude of AC electric field might point the degenerate energy state of FE and AFE orderings. Compared to the HZO FE film, the Rayleigh region is hardly distinguishable for HZZ film and a relatively steep slope $\sim 8$ times higher than that of $\mathrm{HZO}$ in $\epsilon_{\mathrm{r}}$ $E$ graph was observed.

The cross-sectional STEM study collected on HZZ nanolaminate confirmed that the ALD and post-annealing processes had fabricated the $\mathrm{HZO} / \mathrm{ZrO}_{2}$ layer-by-layer structure (Figure 2a). 
Indeed, the gray (HZO layer) and dark $\left(\mathrm{ZrO}_{2}\right.$ layer) regions which present $~ 1$-nm HZO-dominant and $\sim 1$-nm $\mathrm{ZrO}_{2}$-dominant layers, are distinguishable in HAADF-STEM images. It is worth mentioning that the as-deposited amorphous nanolaminate was crystalline through an annealing process at $500{ }^{\circ} \mathrm{C}$ for $30 \mathrm{sec}$; during crystallization (nucleation and growth), inter-diffusion of adjacent layers ( $\mathrm{HZO}$ and $\mathrm{ZrO}_{2}$ ) is inevitable so a sharp interface cannot form. Moreover, we should note that the formation of an interfacial layer between $\mathrm{W}$ and $\mathrm{HZZ}$ is unavoidable since the HZZ layer was deposited at $250^{\circ} \mathrm{C}$ under ozone pulses with a length of $15 \mathrm{sec}$ [29]. Formation of this region is obvious between W and HZZ layers from the STEM image. Especially, the W bottom electrode was substantially affected by fabrication process since the W/HZZ bottom interface was formed during the ALD process. 

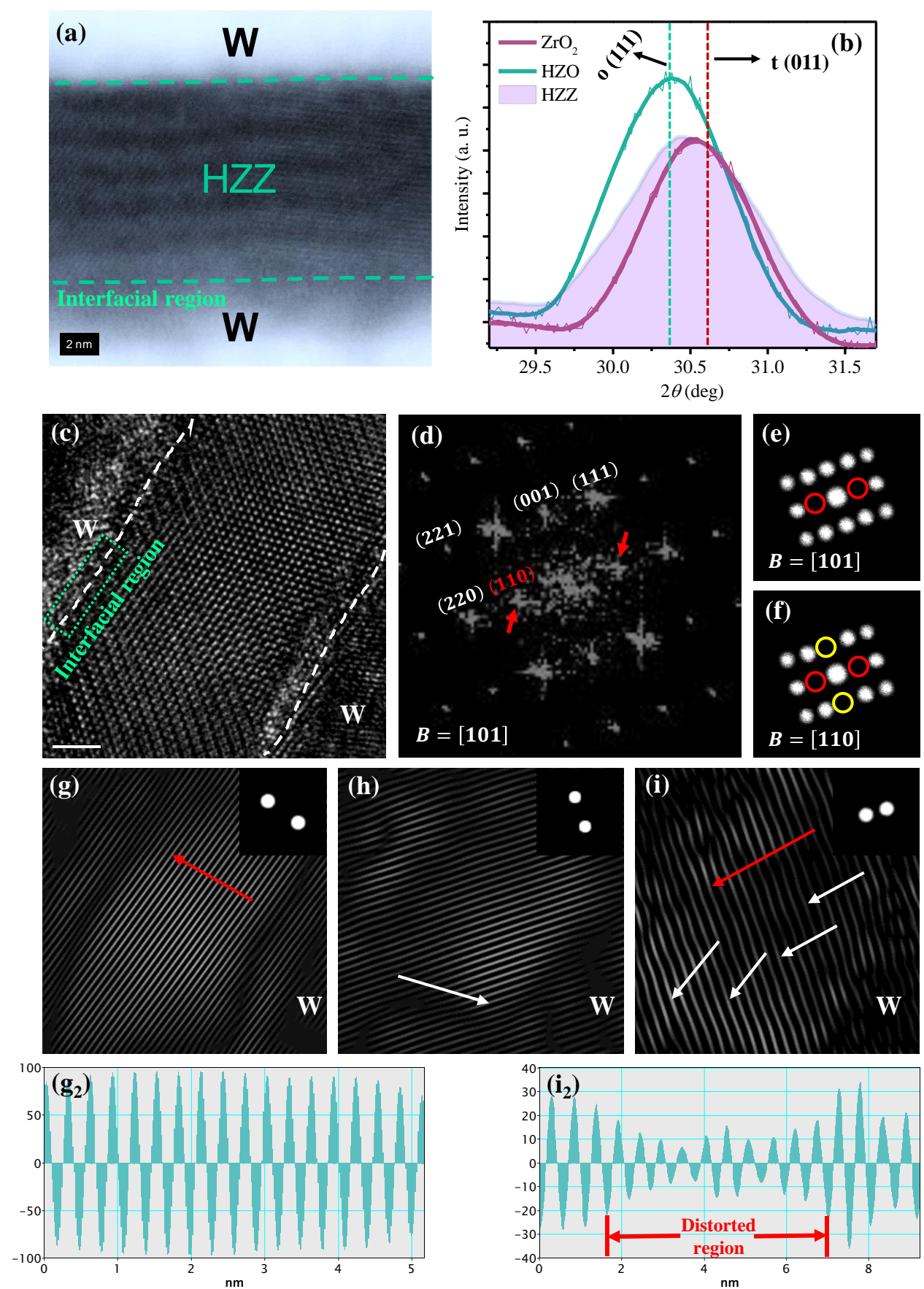

Figure 2. (a) HAADF-STEM cross-sectional image from W/HZZ/W device, (b) XRD patterns of $\mathrm{ZrO}_{2}$, HZO and HZZ films. (c) HRTEM cross-sectional image from W/HZZ/W device, (d) the fast Fourier transform (FFT) pattern of the HZZ layer. SAED diffraction simulations of $P$ ca2 1 phase along the (e) [101] and (f) [110] zone axes. Along the [110] zone, we note that both the (001) and the (1-10) spots are forbidden. Along the [101] zone, (10-1) is forbidden. (g) The filtered (111), (h) (001) and 
(i) (110) inverse fast Fourier transform (IFFT) image. g2 and i2 are the line maps along the red arrows in $\mathrm{g}$ and $\mathrm{i}$, respectively.

The XRD results demonstrate the suppression of monoclinic (m-) phase in all three films (Figure $\mathrm{S} 2$ ). The W capping electrode with relatively low thermal expansion coefficient (TEC) compared to $\mathrm{ZrO}_{2}$ and $\mathrm{HfO}_{2}$ might be responsible for this structural evolution during the annealing process [22]. Electrodes that have relatively low TEC induce in-plane tensile strain during the cooling step of annealing process. The tensile strain may prevent the formation of m-phase by suppression of twin deformation [29]. The XRD patterns show the characteristic o- $\left(2 \theta=30.4^{\circ}\right)$ and t-phase $(2 \theta$ $=30.7^{\circ}$ ) peaks. To enable precise comparison, we obtained XRD patterns focused at a limited range in which the shape of o/t-phase characteristic peaks is most distinguishable (Figure $2 b$ ). The $\mathrm{o} / \mathrm{t}$ XRD characteristic peak of $\mathrm{HZZ}$ nanolaminate lies between those of $\mathrm{HZO}$ and $\mathrm{ZrO}_{2}$ thin films. The $\mathrm{HZO}$ and $\mathrm{ZrO}_{2}$ thin films show symmetric peaks near $2 \theta=30.4^{\circ}$ and $30.7^{\circ}$ which indicate ophase and t-phase dominant structures, respectively. The HZO device had a high $2 P_{\mathrm{r}}$, which represents the formation of a large fraction of o-phase. $P$ - $E$ measurement revealed a pure AFE behavior, which indicates a t-structure in $\mathrm{ZrO}_{2}$ thin film.

The HZZ nanolaminate showed a broad and asymmetric peak at $2 \theta=30.5^{\circ}$, which indicates the coexistence of o- and t-phases. These data confirmed a mixture of FE (from o-phase) and AFE (from t-phase) properties in the HZZ nanolaminate. The broad Bragg peak indicates that the nanolaminate pattern prevents the grain growth and results in a fine crystallite-structured thin film. The asymmetric pattern indicates coexisting t- and o-phase.

However, high resolution transmission electron microscopy (HRTEM) characterization would be a more practical approach to capture any local structural deviation in the HZZ phase caused by either o or $\mathrm{t}$ structures, or combination of them. For that, the HRTEM image of the HZZ nanolaminate along the [101] zone axis is shown in figure $2 \mathrm{c}$, which advocates the well crystallized and homogenous structure of the annealed HZZ layer, surrounded by the two W electrodes. As it was discussed above, it is again worth mentioning that according to our previous study [29] the formation of a dead layer at W/HZZ interface is inevitable as the deposition process was carried out at $250^{\circ} \mathrm{C}$ under 15 -sec ozone pulses. Aygun et al [35] and Tan et al [36] studied the formation of an interfacial layer using the spectroscopic ellipsometer, XRD, Fourier transform infrared, and 
XPS depth profiling techniques. In this work, the formation of interfacial layer could be observed directly through STEM and HRTEM analyses. Since the chemistry of $\mathrm{HfO}_{2}$ and $\mathrm{ZrO}_{2}$ materials are almost the same and the fabrication process of all devices were carried out at the same conditions, i.e. the ALD at $250{ }^{\circ} \mathrm{C}$ under a 15 -sec ozone dosage and the RTA at $500{ }^{\circ} \mathrm{C}$ for $30 \mathrm{sec}$, therefore, we supposed that the formation of interfacial layers between $\mathrm{W}$ and $\mathrm{HfO}_{2}$ or $\mathrm{ZrO}_{2}$ are almost the same and the dielectric properties of each device were affected by this region equally.

The co-existence of the both o and t phases can be deducted based on the observation of extra (110) spots (red arrows) in the fast Fourier transform (FFT) pattern of the HZZ layer in figure 2d which can be further assessed as follows.

Firstly, in order to investigate the importance of the applied zone axis in HRTEM characterization, the selected area diffraction (SAED) simulation of $P \mathrm{ca} 2_{1}$ phase, along the [101] and [110] zone axes are shown in figure 2e and f, respectively [37]. Along the [110] axis in figure $2 \mathrm{f}$ both (001) and (1-10) spots are absent, which are indicated by yellow and red open circles, respectively. Along the [101] zone in figure 2e, (10-1) is forbidden. Therefore, in the case of either [110] or [101], the FFT pattern in figure $2 \mathrm{~d}$ is clearly suggesting that we have another phase coexisting alongside with the commonly reported $P \mathrm{ca} 2{ }_{1}$ phase, which is nothing but t-phase.

For more clarification, the (111) filtered inverse fast Fourier transform (IFFT) image represented in figure $2 \mathrm{~g}$. The line map captured along the red arrow well shows the absence of any kind of distortion in the (111) planes (Figure $2 \mathrm{~g}_{2}$ ). While a local distortion may be noticed along the white arrow in figure $2 \mathrm{~h}$ when the same condition is governed for the (001) planes, these atomic planes have not experience any distortion, which implies the fact that the applied zone axis must be [101] as is shown in SAED simulation pattern in figure 2e. However, the situation is completely different for (110) planes in the HZZ layer. As is obvious in figure 2i, (110) atomic planes of the HZZ layer are extremely distorted along the drawn arrows, which is consistent with the captured line map along the red arrow in figure $2 \mathrm{i}_{2}$.

To optimize the fabrication condition, the effect of post-annealing processes on the evolution of structural features, and the dielectric and FE properties was investigated.

$P$-E curves (Figure 3a) were obtained from HZZ nanolaminates annealed under different conditions. Annealing for $30 \mathrm{~s}$ at annealing temperature $T_{\mathrm{ANN}}>350^{\circ} \mathrm{C}$ caused the appearance of 
FE-AFE mixed loop. Below $350{ }^{\circ} \mathrm{C}$ the film showed a linear dielectric behavior (data was not presented here.).

(a)

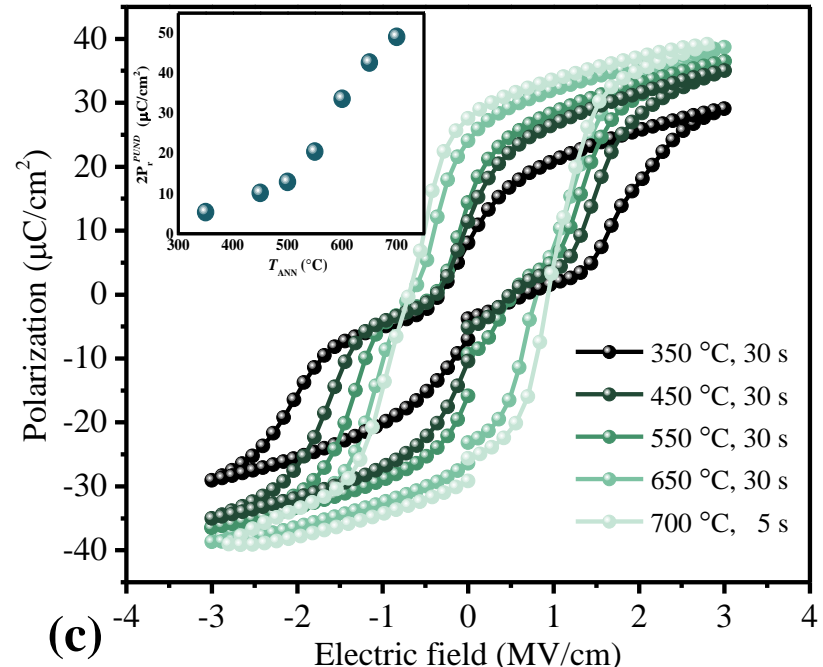

(b)

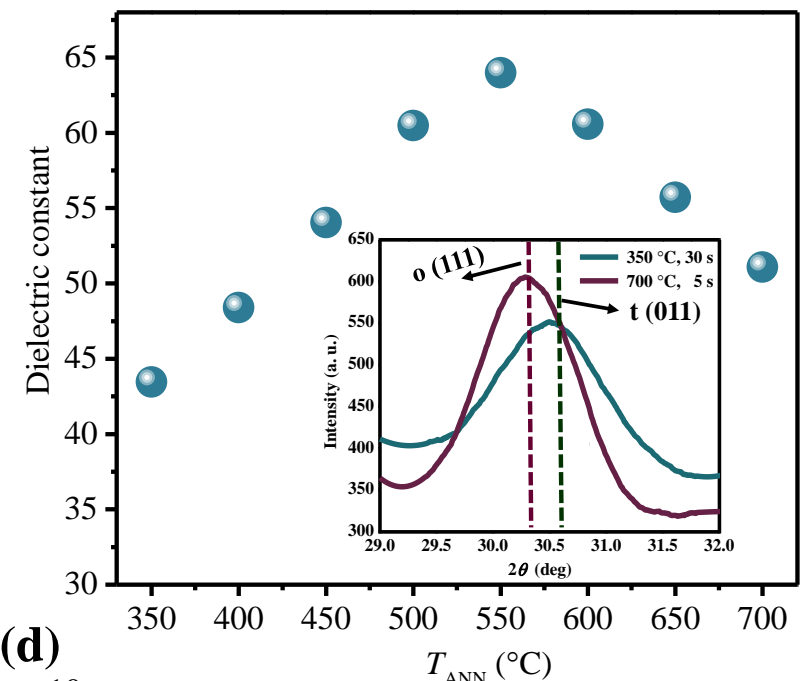

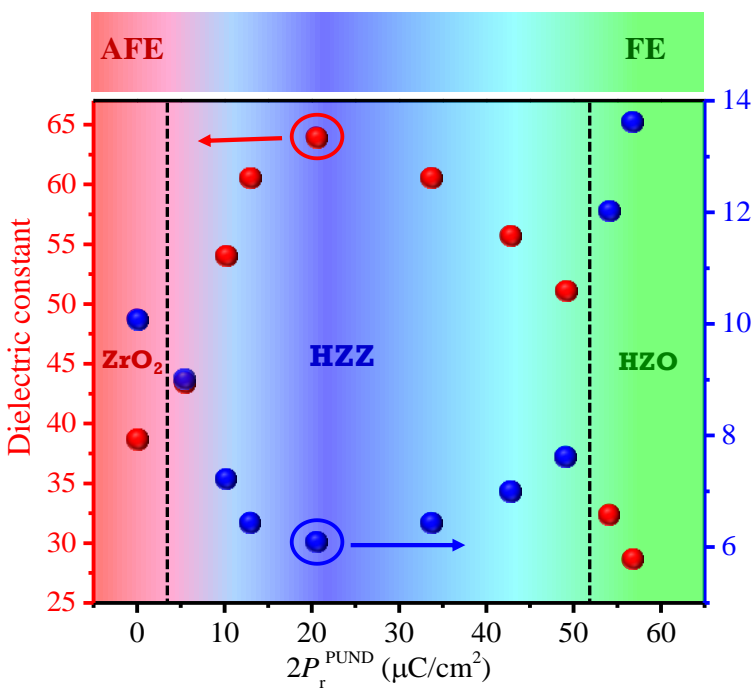

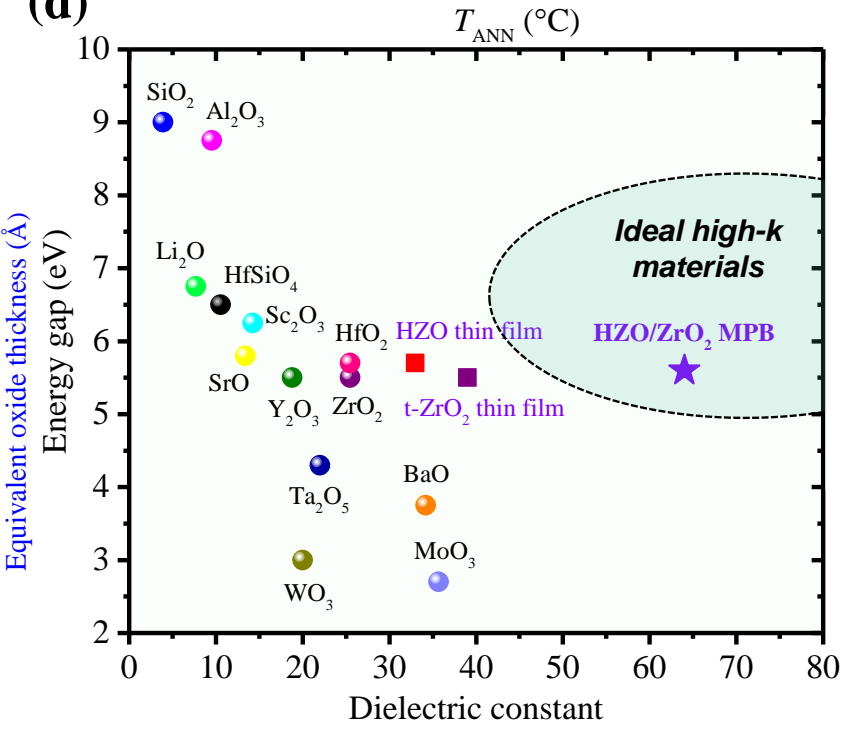

Figure 3. (a) P-E loops for the $\mathrm{W} / \mathrm{HZZ} / \mathrm{W}$ devices annealed at different temperatures $T_{\mathrm{ANN}}$ for $30 \mathrm{~s}$. Annealing was performed at $700{ }^{\circ} \mathrm{C}$ for $5 \mathrm{~s}$ to avoid aggressive reaction at the dielectric/metal interface. Inset: $2 P_{r}^{P U N D}$ for HZZ device annealed at different $T_{\mathrm{ANN}}$. (b) Effect of $T_{\mathrm{ANN}}$ on the dielectric constant of W/HZZ/W devices. (c) Dielectric constant (red spheres) and EOT (blue spheres) versus $2 P_{r}^{P U N D}$ values of $\mathrm{HfO}_{2}-\mathrm{ZrO}_{2}$ thin film with different design. (d) Experimentally-measured band gap versus dielectric constant of well-known oxides extracted from reference [5]. The present work (purple star) achieved an ideal 
dielectric constant of $\mathrm{HfO}_{2}-\mathrm{ZrO}_{2}$ through MPB design. It was supposed that the nanolaminate design doesn't affect the band gap of $\mathrm{HfO}_{2}$ and $\mathrm{ZrO}_{2}$ materials.

Increase in $T_{\mathrm{ANN}}$ widened the hysteresis loops, and caused an increase in $2 P_{\mathrm{r}}$ and a gradual disappearance of the double-hysteresis characteristics. Therefore, AFE-dominant behavior changed to FE-dominant behavior as $T_{\mathrm{ANN}}$ increased. $2 P_{\mathrm{r}}$ substantially increased from 5.4 to $48.8 \mu \mathrm{C} / \mathrm{cm}^{2}$ as $T_{\mathrm{ANN}}$ was increased from $350{ }^{\circ} \mathrm{C}$ to $700{ }^{\circ} \mathrm{C}$; the change can be attributed to an increase in the fraction of o-phase due to the intermixing of $\mathrm{HfO}_{2}$ and $\mathrm{ZrO}_{2}$ at the interfaces during the annealing process. Moreover, as $T_{\mathrm{ANN}}$ is increased, the grain size increases, and may impede the stabilization of t-phase after cooling to RT. In fact, the o-phase is stable at medium grain sizes between $\mathrm{t}$ - and m-phases. The surface energy of o-phase is $2.575 \mathrm{~J} / \mathrm{cm}^{2}$, whereas it is $2.5 \mathrm{~J} / \mathrm{cm}^{2}$ for $\mathrm{t}$-phase and $3.2 \mathrm{~J} / \mathrm{cm}^{2}$ for $\mathrm{m}$-phase [38]. An increased in-plane tensile strain induced by the $\mathrm{W}$ top and bottom electrode can provide additional force to drive the transition from t-phase to o-phase by annealing at high $T_{\mathrm{ANN}}$. In the XRD pattern of HZZ nanolaminates, the o/t characteristic peak of the structure was left-shifted after $T_{\mathrm{ANN}}=700{ }^{\circ} \mathrm{C}$ compared to $T_{\mathrm{ANN}}=350{ }^{\circ} \mathrm{C}$ (figure $3 \mathrm{~b}$ ); this change indicates an increase in the o-phase fraction throughout the film after annealing at $T_{\mathrm{ANN}}=$ $700{ }^{\circ} \mathrm{C}$. As $T_{\mathrm{ANN}}$ increased, FWHM of the o/t characteristic peak decreased; the change is attributed to improved crystallization driven by the thermal energy. The t-phases are stable at the smallest crystallite sizes. Therefore, a combination of left-shift and broadened Bragg peak revealed that dominance of t-phase in the films increased as $T_{\mathrm{ANN}}$ decreased.

A temperature at which the maximum $\rho_{M P B}$ can be achieved is promising for exceptionally high$\kappa$ device. Increasing $T_{\mathrm{ANN}}$ from $350{ }^{\circ} \mathrm{C}$ to $550{ }^{\circ} \mathrm{C}$ caused an increase in $\epsilon_{\mathrm{r}}$. Annealing at $550{ }^{\circ} \mathrm{C}$ for $30 \mathrm{sec}$ gave the maximum $\epsilon_{\mathrm{r}}=64$, which may suggest that this condition yielded the maximum $\rho_{M P B}$ in $\mathrm{HZZ}$ nanolaminate. $T_{\mathrm{ANN}}>550^{\circ} \mathrm{C}$ caused a reduction in $\epsilon_{\mathrm{r}}$. Therefore, annealing at $T_{\mathrm{ANN}}$ either higher than or lower than $550{ }^{\circ} \mathrm{C}$ caused a decrease in $\epsilon_{\mathrm{r}}$ of the $\mathrm{HZZ}$ nanolaminate, possibly as a result of increase in formation of o- and t-phase, respectively. Consequently, the $\rho_{M P B}$ can decrease. $2 P_{\mathrm{r}}$ were measured using positive-up-negative-down (PUND) method to correct for the effect of dielectric loss on the measured polarization. Increase in $T_{\mathrm{ANN}}$ causes an increase in the leakage current of $\mathrm{W} / \mathrm{HZZ} / \mathrm{W}$ device by inducing formation of interfacial layers at the W/HZZ interface that can extract oxygen from HZZ layers [29]. The PUND method is helpful to subtract the effect of leakage current from the measured polarization. As $2 P_{\mathrm{r}}$ increased, $\epsilon_{\mathrm{r}}$ first increased to 
a maximum of 64 at $2 P_{\mathrm{r}} \approx 20 \mu \mathrm{C} / \mathrm{cm}^{2}$, then decreased (Figure $3 \mathrm{c}$ ). This result may mean that $2 P_{\mathrm{r}}$ $\approx 20 \mu \mathrm{C} / \mathrm{cm}^{2}$, induces the highest $\rho_{M P B}$ at the interfaces of $\mathrm{HZO}(\mathrm{FE})-\mathrm{ZrO}_{2}$ (AFE) nanolaminates. For comparison, the graph includes the dielectric properties of $\mathrm{ZrO}_{2}\left(2 P_{\mathrm{r}}=0\right)$ and $\mathrm{HZO}\left(2 P_{\mathrm{r}}=54\right.$ $\mu \mathrm{C} / \mathrm{cm}^{2}$ (annealed at $500{ }^{\circ} \mathrm{C}$ for $30 \mathrm{~s}$ ) and $57 \mu \mathrm{C} / \mathrm{cm}^{2}$ (annealed at $700{ }^{\circ} \mathrm{C}$ for $5 \mathrm{~s}$ )) at the ends. The $\mathrm{ZrO}_{2}$ thin film with $2 P_{\mathrm{r}}=0$ gives a $\epsilon_{\mathrm{r}}$ of 39 and a pure $\mathrm{HZO}$ film shows $28 \leq \epsilon_{\mathrm{r}} \leq 32$ depending on the annealing conditions. A nanolaminate structure annealed at an appropriate condition had a dielectric constant $73 \%$ higher than that of $\mathrm{ZrO}_{2}$ and $\mathrm{HZO}$ films. The EOT measurements showed a reduction to $\sim 6 \AA$. Assuming that the nanolaminate design does not affect $E_{\mathrm{g}}$ of either parent phases $\left(\mathrm{ZrO}_{2}, \mathrm{HfO}_{2}\right)$ then $E_{\mathrm{g}}$ as a function of $\epsilon_{\mathrm{r}}$ (figure $3 \mathrm{~d}$ ) shows that the new design is a highly promising approach towards the production of ideal high- $\kappa$ materials.

\section{Conclusion}

The dielectric properties of $\mathrm{HfO}_{2}-\mathrm{ZrO}_{2}$ based thin film was enhanced through $\mathrm{HZZ}$ nanolaminate design. While the $\mathrm{HZO}$ and $\mathrm{ZrO}_{2}$ thin films showed pure FE and AFE features, respectively, the HZZ nanolaminate revealed a complex P-E loop consists of both FE and AFE characteristics. The unusual large $\epsilon_{\mathrm{r}}$ of $\mathrm{HZZ}$ nanolaminate compared to the $\mathrm{HZO}$ and $\mathrm{ZrO}_{2}$ films was attributed to the formation of MPB region. In fact, the P-E curve showed a combination of FE and AFE characteristics which may introduce MPB regions throughout the nanolaminate structure. The coexistence of $t$ and o phases was confirmed through XRD and HRTEM studies and the nature of nanolaminate structure was confirmed by STEM analysis. To increase the formation of MPB region inside the $\mathrm{HZZ}$ nanolaminate, the annealing condition was adjusted. A high $\epsilon_{\mathrm{r}}$ of 64 was achieved after an appropriate annealing process. This work presents a new design to increase the dielectric properties of $\mathrm{HfO}_{2}-\mathrm{ZrO}_{2}$ based thin film. The fabrication process is performed at a low thermal budget $\left(250^{\circ} \mathrm{C}\right)$ and the device is compatible with silicon technology, so the new design introduces a promising device for future electronics.

\section{Acknowledgements}

The authors thank Mr. Mehrdad Ghiasabadi Farahani, Dr. Stanislav Kamba, Dr. Kai Ni, Dr. Writam Banerjee, Dr. Nikam and Mr. Peyman Asghari-Rad for helpful discussions. This work was supported by the National Research Foundation of Korea funded by the Korean government (MSIT), grant no. NRF-2018R1A3B1052693. 


\section{Contributions}

A. Kashir conceived the idea, performed the experiments, analyzed the results and wrote the manuscript. H. Hwang supervised the project and reviewed the manuscript.

\section{References}

1. Kim, S. K. et al. Capacitors with an equivalent oxide thickness of $<0.5 \mathrm{~nm}$ for nanoscale electronic semiconductor memory. Adv. Funct. Mater. 20, 2989-3003 (2010).

2. Kim, S. K. et al. Al-doped $\mathrm{TiO}_{2}$ films with ultralow leakage currents for next generation DRAM capacitors. Adv. Mater. 20, 1429-1435 (2008).

3. Jeon, W. et al. Evaluating the top electrode material for achieving an equivalent oxide thickness smaller than $0.4 \mathrm{~nm}$ from an Al-doped $\mathrm{TiO}_{2}$ film. ACS Appl. Mater. Interfaces 6, 21632-21637 (2014).

4. Lee, S. W. et al. Atomic layer deposition of $\mathrm{SrTiO}_{3}$ thin films with highly enhanced growth rate for ultrahigh density capacitors. Chem. Mater. 23, 2227-2236 (2011).

5. Yim, K. et al. Novel high-K dielectrics for next-generation electronic devices screened by automated ab initio calculations. NPG Asia Mater. 7, 1-6 (2015).

6. Pignedoli, C. A. et al. Anomalous behavior of the dielectric constant of hafnium silicates: A first principles study. Phys. Rev. Lett. 98, 1-4 (2007).

7. Tomida, K. et al. Dielectric constant enhancement due to $\mathrm{Si}$ incorporation into $\mathrm{HfO}_{2}$. Appl. Phys. Lett. $89,2-5$ (2006).

8. Wiemer, C. et al. Dielectric properties of Er- doped $\mathrm{HfO}_{2}(\mathrm{Er} \sim 15 \%)$ grown by atomic layer deposition for high- $\kappa$ gate stacks. Appl. Phys. Lett. 96, 1-4 (2010).

9. Böscke, T. S. et al. Increasing permittivity in $\mathrm{HfZrO}$ thin films by surface manipulation. Appl. Phys. Lett. 95, 2-5 (2009).

10. Cho, H. J. et al. New TIT capacitor with $\mathrm{ZrO}_{2} / \mathrm{Al}_{2} \mathrm{O}_{3} / \mathrm{ZrO}_{2}$ dielectrics for $60 \mathrm{~nm}$ and below DRAMs. Solid. State. Electron. 51, 1529-1533 (2007).

11. Kil, D. S. et al. Development of new $\mathrm{TiN} / \mathrm{ZrO}_{2} / \mathrm{Al}_{2} \mathrm{O}_{3} / \mathrm{ZrO}_{2} / \mathrm{TiN}$ capacitors extendable to $45 \mathrm{~nm}$ generation DRAMs replacing $\mathrm{HfO}_{2}$ based dielectrics. Dig. Tech. Pap. - Symp. VLSI Technol. 65, 38-39 (2006).

12. Zhang, X. Y. et al. Temperature-Dependent $\mathrm{HfO}_{2} / \mathrm{Si}$ Interface Structural Evolution and its Mechanism. Nanoscale Res. Lett. 14, 0-7 (2019).

13. Choi, K.-J. et al. Control of the Interfacial Layer Thickness in Hafnium Oxide Gate Dielectric Grown by PECVD. J. Electrochem. Soc. 150, F75 (2003).

14. Ni, K. et al. Equivalent Oxide Thickness (EOT) Scaling with Hafnium Zirconium Oxide High-א Dielectric Near Morphotropic Phase Boundary. Tech. Dig. - Int. Electron Devices Meet. IEDM 2019Decem, 7-10 (2019). 
15. Ahart, M. et al. Origin of morphotropic phase boundaries in ferroelectrics. Nature 451, 545-548 (2008).

16. Cross, E. Lead-free at last. Nature 432, 24-25 (2004).

17. Guo, R. et al. Origin of the high piezoelectric response in $\mathrm{PbZr}_{1-\mathrm{x}} \mathrm{Ti}_{\mathrm{x}} \mathrm{O}_{3}$. Phys. Rev. Lett. 84, 54235426 (2000).

18. Fu, H. et al. Polarization rotation mechanism for ultrahigh electromechanical response in singlecrystal piezoelectrics. Nature 403, 281-283 (2000).

19. Kutnjak, Z. et al.The giant electromechanical response in ferroelectric relaxors as a critical phenomenon. Nature 441, 956-959 (2006).

20. Zheng, T. et al. The structural origin of enhanced piezoelectric performance and stability in lead free ceramics†. Energy Environ. Sci. 10, 528-537 (2017).

21. Dogan, M. et al. Causes of ferroelectricity in $\mathrm{HfO}_{2}$-based thin films: An: ab initio perspective. Phys. Chem. Chem. Phys. 21, 12150-12162 (2019).

22. Cao, R. et al. Effects of Capping Electrode on Ferroelectric Properties of $\mathrm{Hf}_{0.5} \mathrm{Zr}_{0.5} \mathrm{O}_{2}$ Thin Films. IEEE Electron Device Lett. 39, 1207-1210 (2018).

23. Batra, R. et al. Factors Favoring Ferroelectricity in Hafnia: A First-Principles Computational Study. J. Phys. Chem. C 121, 4139-4145 (2017).

24. Müller, J. et al. Ferroelectricity in simple binary $\mathrm{ZrO}_{2}$ and $\mathrm{HfO}_{2}$. Nano Lett. 12, 4318-4323 (2012).

25. Huan, T. D. et al. Pathways towards ferroelectricity in hafnia. Phys. Rev. B - Condens. Matter Mater. Phys. 90, 1-5 (2014).

26. Park, M. H. et al. Morphotropic Phase Boundary of Hf 1- x Zr x O 2 Thin Films for Dynamic Random Access Memories. ACS Appl. Mater. Interfaces 10, 42666-42673 (2018).

27. Reyes-Lillo, S. E. et al. Antiferroelectricity in thin-film $\mathrm{ZrO}_{2}$ from first principles. Phys. Rev. B Condens. Matter Mater. Phys. 90, 1-5 (2014).

28. Sang, X. et al. On the structural origins of ferroelectricity in $\mathrm{HfO}_{2}$ thin films. Appl. Phys. Lett. 106, 162905 (2015).

29. Kashir, A. et al. Large Remnant Polarization in a Wake-Up Free Hf0. 5Zr0. 5O2 Ferroelectric Film through Bulk and Interface Engineering. ACS Applied Electronic Materials (2021).

30. Cheema, S. S. et al. Enhanced ferroelectricity in ultrathin films grown directly on silicon. Nature 580, 478-482 (2020).

31. Bintachitt, P. et al. Collective dynamics underpins Rayleigh behavior in disordered polycrystalline ferroelectrics. Proc. Natl. Acad. Sci. U. S. A. 107, 7219-7224 (2010).

32. $\mathrm{Xu}, \mathrm{R}$. et al. Stationary domain wall contribution to enhanced ferroelectric susceptibility. Nat.

Commun. 5, 1-7 (2014).

33. Bassiri-Gharb, N. et al. Domain wall contributions to the properties of piezoelectric thin films. J.

Electroceramics 19, 47-65 (2007). 
34. Grimley, E. D. et al. Structural Changes Underlying Field-Cycling Phenomena in Ferroelectric $\mathrm{HfO}_{2}$ Thin Films. Advanced Electronic Materials, 2, 1600173 (2016).

35. Aygun, G. et al. Interfacial and structural properties of sputtered HfO 2 layers. Journal of Applied Physics, 106(1), p.014312. (2009).

36. Tan, R. et al. Comparative study of the interfacial characteristics of sputter-deposited HfO2 on native $\mathrm{SiO} 2 / \mathrm{Si}$ (100) using in situ XPS, AES and GIXR. Surface and Interface Analysis: An International Journal devoted to the development and application of techniques for the analysis of surfaces, interfaces and thin films, 38(4), pp.784-788. (2006).

37. Wei, Yingfen, et al. A rhombohedral ferroelectric phase in epitaxially strained $\mathrm{Hf}_{0.5} \mathrm{Zr}_{0.5} \mathrm{O}_{2}$ thin films." Nature materials 17, 1095, (2018).

38. Materlik, R. et al. The origin of ferroelectricity in $\mathrm{Hf}_{1-\mathrm{x}} \mathrm{Zr}_{\mathrm{x}} \mathrm{O}_{2}$ : A computational investigation and a surface energy model. J. Appl. Phys. 117, 134109 (2015). 